\title{
LA RESPONSABILIDAD SOCIAL CORPORATIVA DESDE EL ENFOQUE DE LAS RELACIONES PÚBLICAS: ESTRATEGIA DE GESTIÓN RELACIONAL EN EL CONTEXTO DEL DESARROLLO LOCAL Y TURISMO SOSTENIBLE
}

\author{
Antonia Pérez-García \\ Universidade da Coruña-Escuela Universitaria de Turismo \\ antonia.perezg@udc.es
}

Submetido 22/10/2016 - Aceito 11/11/2017

DOI: 10.15628/holos.2017.5244

\section{RESUMEN}

La responsabilidad social corporativa es capital en la gestión de cualquier empresa u organización, máxime si estamos hablando de una institución pública. Cultivar y ejecutar la responsabilidad social para crear imagen de transparencia y compromiso, debe de ser, y es, uno de los objetivos más claros e importantes a la hora de trabajar los proyectos de desarrollo local turístico sostenible. Las organizaciones e instituciones esperan que con este compromiso, adoptado voluntariamente, contribuya a incrementar su imagen y rentabilidad, además de atraer un turista más responsable. El objetivo de este artículo de revisión será abordar la responsabilidad social corporativa como una de las premisas y estrategias de gestión, desde la disciplina de las relaciones públicas, con la idea de proponer una línea de gestión relacional en las políticas de desarrollo local sustentable y más responsable en el contexto turístico.

PALAVRAS-CHAVE: Responsabilidad Social Corporativa, Relaciones Públicas, Turismo, Desarrollo Local Sostenible.

\section{CORPORATE SOCIAL RESPONSIBILITY FROM THE PUBLIC RELATIONS PERSPECTIVE: RELATIONAL MANAGEMENT STRATEGY IN THE CONTEXT OF LOCAL DEVELOPMENT AND SUSTAINABLE TOURISM}

\begin{abstract}
Corporate social responsibility is central to the management of any company or organization, but this is especially true in the case of public institutions. Cultivating and implementing social responsibility to create an image of transparency and commitment, should be, and need is, one of the clearest key objectives when working on local sustainable tourism development projects. Organizations and institutions expect that this commitment, entered into on a
\end{abstract}

voluntary basis, will help to increase their image and profitability and attract a more responsible tourist. The aim of this review article is to address corporate social responsibility as a management premise and strategy from the perspective of public relations, in order to propose a line of relational management policies sustainable and more responsible local development policies within the context tourism.

KEYWORDS: Corporate Social Responsibility, Public Relations, Tourism, Sustainable Local. 


\section{INTRODUCCIÓN}

La actividad turística se presenta como una oportunidad para el desarrollo local y, en concreto, el turismo sustentable se manifiesta como una vía de mejora de la calidad de vida de la población local ya que respeta el medio natural, la cultura local, a la vez que impulsa los recursos endógenos del propio municipio. El aprovechamiento de los recursos naturales y culturales del municipio es la idea que subyace en el desarrollo del turismo sostenible local, esta idea se puede ver reforzada a través de la estrategia de la gestión relacional de la responsabilidad social corporativa. Por lo que el presente artículo de revisión teórica trata de presentar una propuesta de gestión desde el enfoque filosófico de las relaciones públicas.

\section{RESPONSABILIDAD SOCIAL CORPORATIVA}

El término de "responsabilidad social corporativa" (RSC) está vinculado al significado que tiene que ver con la ética de cómo hacer bien las cosas ante los diferentes públicos, por lo que conectaríamos con la máxima de actuación en relaciones públicas (RR.PP): "Hacerlo bien y hacerlo saber", de la cual aclara Noguero (1990) "(...) se implican los factores de producción con sus características técnicas y humanas (...) a partir del medio interno, se estudiará y ejecutará una política de imagen pública a través de unas actuaciones concretamente objetivas y reales" (p. 50). Esta dimensión ética es primordial en el mundo actual ya que podemos observar una extendida carencia de sentimientos, de actitudes generosas, solidarias y altruistas por parte de las empresas, por lo que, aquellas que sí demuestran esta preocupación por la responsabilidad social tendrán mayor éxito en sus proyectos. Por otro lado, se destaca el hecho de que el concepto de "responsabilidad social" también estaría coaligado, o incluso englobaría otros tipos de responsabilidades como son las jurídicas, políticas, civiles, penales y contractuales; porque la responsabilidad social es mucho más amplia.

Retomando la idea de la ética relacionada con la comunicación empresarial, dirá Weil P. (1992) "Cuando la empresa habla se compromete y debe mantener con honor su palabra. En caso contrario, perderá su legitimidad, su credibilidad, su autoridad, y esto no le traerá nada positivo" (p. 154). Esta visión ha de impregnar a toda la organización en su totalidad, es una toma de conciencia como sujeto jurídico y no debe de estar sujeto a modas o presentarse como un parche ante una situación de crisis. Weil afirma que esta ética debe suponer un "valor añadido" a la empresa y que constituye:

- La identidad de la empresa que ha de inspirar todos sus actos, gestión y productos.

- Una fuente de fuerza y de autonomía frente a la competencia y un medio para evitar caer en la banalidad del discurso.

- Una meta y un estímulo de trabajo de todas las áreas de la empresa

- El hilo que guía todos los actos de la empresa que debe originar respeto y apego.

- Permitirá establecer con los públicos una relación que va más allá de lo meramente comercial, es como un acuerdo civil. 
Algunos autores como Seitel, F. (2002) identifican el viejo concepto de filantropía con el más reciente de responsabilidad social. Parés i Maicas (1994) aclara que el concepto de filantropía también se asocia a los conceptos de mecenazgo, fundación y patrocinio pero con limitaciones conceptuales ya que la filantropía debe ser entendida de diferente forma porque va más allá, se entiende como la aportación de riqueza privada a objetivos públicos. Otros autores, en cambio, afirman que las actuaciones filantrópicas son en la actualidad una pequeña parte de las estrategias de la RSC, ya que ésta abarca una nueva dimensión; Wilcox y otros (2012) dirán:

Otra manifestación de la responsabilidad social corporativa es la filantropía corporativa, la donación de fondos, productos y servicios a causas diversas (...). Las corporaciones, por supuesto, han utilizado la filantropía desde hace mucho para demostrar a la comunidad su buena voluntad y pulir su reputación de buenos ciudadanos. (p. 487)

De acuerdo con Carroll (1991), citado por Balaguer Franch, M.R. y otros (2007), la RSC tendría un significado más amplio por lo que propone un modelo conceptual donde incluye una variedad de responsabilidades de la empresa junto a la sociedad y aclara que los componentes de la responsabilidad social empresarial han de ir más allá de generar beneficios y obedecer la ley. El modelo englobaría cuatro tipos básicos que Carroll plantea de forma piramidal por orden de importancia:

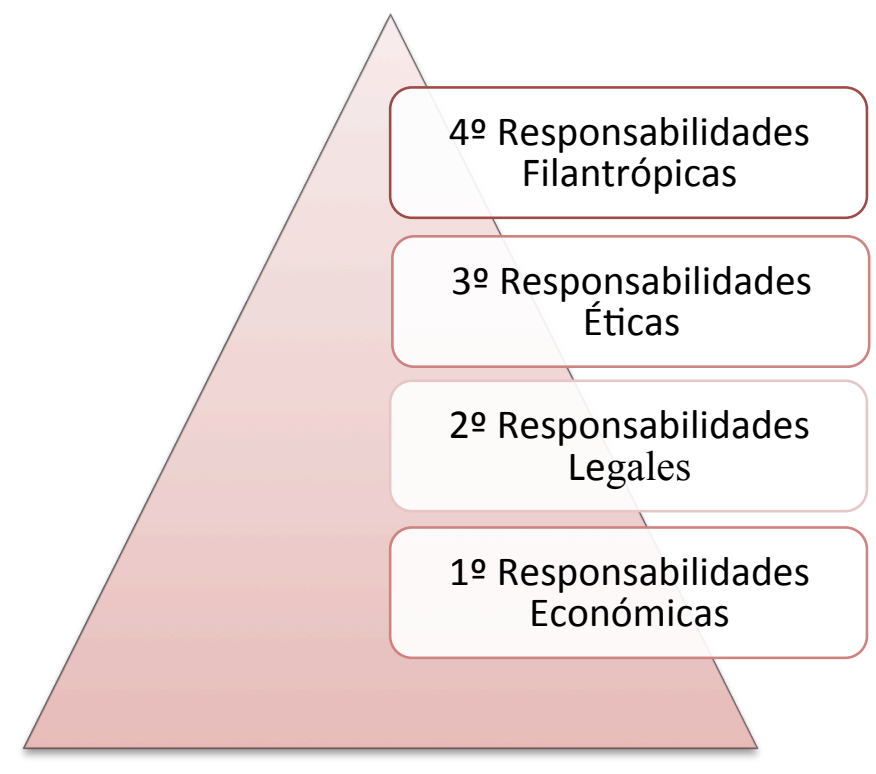

Figura 1: Pirámide Responsabilidad Social de Carroll.

(1ํ) Responsabilidades Económicas, serían las primeras a tener en cuenta porque, según Carroll, lo más importante es producir bienes y servicios para poder venderlos y obtener beneficios que mantengan el sistema capitalista.

(2) Las Responsabilidades Legales, es decir, obedecer la ley serían las segundas ya que la sociedad espera que las empresas actúen dentro de la legalidad establecida por los gobiernos, ya sea en temática de conservación del medio ambiente o protección al consumidor.

(3ㅇ) Responsabilidades Éticas que sería la obligación de hacer lo correcto y lo justo por parte de las empresas y estarían en el tercer lugar en orden de importancia. 
(4ㅇ) Finalmente estarían las Responsabilidades Filantrópicas, o Sociales como contribuir a mejorar la vida de la comunidad aportando recursos. Estas responsabilidades no son obligatorias para las empresas y estarían guiadas por el deseo de ejercer un papel de aportación de beneficio social a la comunidad.

\section{RESPONSABILIDAD SOCIAL Y RELACIONES PÚBLICAS}

Durante la segunda mitad de la década de los ochenta y la primera de los noventa aparecen una gran cantidad de investigaciones y publicaciones sobre la responsabilidad social, muchas de ellas ligadas a la disciplina de las relaciones públicas, suponiendo una aportación importante al ámbito de la misma.

Un antecesor a todos ellos y precursor a la hora de aproximarse a este tema, desde la óptica de las relaciones públicas, fue Bernays, E. que en su obra Crystallizing Public Opinion de 1923, llega a afirmar sobre este tema:

El consultor de relaciones públicas ha de someter sus acciones a un intenso escrutinio, para evitar la propagación de movimientos o ideas antisociales o peligrosas. El valor social del consultor de relaciones públicas reside en el hecho que lleva a los públicos hechos e ideas de utilidad social que, de otra forma, no lograrían una adecuada aceptación (...) el consultor de relaciones públicas está destinado a la creación de la conciencia pública (...). (Bernays, E., 1990: 23-24)

Parafraseando a Black, S. (1991) éste diría al respecto, que la idea de responsabilidad social, por parte de la empresa, es que su deber es permanecer fuerte y eficiente de forma que pueda seguir contribuyendo a sus públicos, accionistas y empleados, pero al mismo tiempo tiene que hacer una contribución fundamental a la economía y al bienestar del país. La RSC abarcaría, de forma práctica, una serie de categorías o temas de interés social como son:

- Empresa: Con el apoyo y el desarrollo de iniciativas para promocionar a los jóvenes empresarios, y de esta forma impulsar la prosperidad de la empresa.

- Enseñanza: Ayudando a crear nuevas metas en la vida de la gente joven.

- Arte y cultura: Colaborando con las iniciativas y actividades artísticas y de proximidad de las comunidades.

- Medio Ambiente: Estimulando los esfuerzos para sostener y proteger el medio ambiente y mejorar la calidad de vida de los ciudadanos, es decir, de la comunidad.

Sobre esta última categoría, Black, S. (1993) destaca el hecho de que en estos asuntos también tome el control el profesional de relaciones públicas, no solo la dirección o gerencia ya que la protección al medio ambiente es una responsabilidad conjunta de todos y es esencial explicar correctamente la situación a todos los públicos. Por otro lado, ayudar a la comunidad no se debe de entender como un simple hecho de altruismo por parte de la empresa, sino como un egoísmo bien entendido porque existen unos claros beneficios para la empresa que ejecuta o ejerce su labor en una comunidad amistosa. Black establece una sencilla guía de política empresarial al respecto de estos asuntos, desde la óptica de las relaciones públicas: 
1. Hay que seleccionar los problemas o asuntos conflictivos que supongan un nivel alto de preocupación local o nacional.

2. Hay que concentrar los esfuerzos en unas áreas concretas donde su impacto y visibilidad sean más altos.

3. Hay que utilizar técnicas eficaces para comunicar y divulgar los proyectos sociales y comunitarios de la empresa y, por supuesto, también sus éxitos.

4. Hay que reducir, en la medida de lo posible, los objetivos que sean solo de interés corporativo porque se pueden malinterpretar por parte de la comunidad.

Según Grunig y Hunt (2000) la RSC se puede clasificar como la realización de tareas y programas que denoten la preocupación de la organización por solucionar problemas sociales generales no conectados con la misma; pero estos problemas sociales, confirman, son muy amplios por ello proponen diferenciar responsabilidad pública de responsabilidad social. Siguiendo a Preston y Post (1975), citados por ellos mismos, inciden en afirmar que la responsabilidad social no tiene límites y que la organización no puede saber dónde empiezan y acaban estas responsabilidades por lo que es mejor utilizar el criterio de "responsabilidad pública" porque le otorga a la empresa mayor información sobre sus responsabilidades al atender a diversas consecuencias de implicaciones de tipo "primario o secundario". En este caso, está claro que la empresa debería ejercer las relaciones públicas, y lo que puede hacer es informar a los subsistemas de la empresa sobre lo que "los públicos creen que son conductas irresponsables de esa organización. También puede informar a los públicos de lo realizado responsable o irresponsablemente por la organización" (Grunig y Hunt: 119). Apuntan a la necesidad de realizar informes o balances internos y externos de forma que se pueda informar sobre las consecuencias económicas y sociales, la clave estaría en el papel del profesional de las relaciones públicas que pudiese ejercer una acción de vigilante, controlador y comunicador de la responsabilidad pública.

Vemos entonces que la responsabilidad social corporativa puede afectar a todos los niveles de la actividad empresarial y está íntimamente relacionada con la conducta ética de dichas actividades. Otro autor que elabora una lista de categorías a tener en cuenta, desde el punto de vista de la dirección de la organización, es Fraser S. (2002) el cual llega a establecer hasta un total de ocho niveles de responsabilidades, entre las cuales estaría la importante gestión relacional:

- Líneas de productos: En cuanto a rendimiento, estándares, productos nocivos, embalaje o impacto medioambiental de los mismos.

- Prácticas de marketing: En cuanto a ventas, reclamaciones, contenido de los anuncios o la política de precios justos de los productos o servicios.

- Filantropía empresarial: En cuanto a contribuciones, proyectos sociales o actividades de desarrollo a la comunidad local.

- Actividades ecológicas: En cuanto al control de la contaminación, control de calidad de los productos y embalajes y/o servicios contaminantes.

- Relaciones Externas: En cuanto a las acciones de apoyo a minorías o relaciones institucionales.

- Promoción del empleo para minorías y mujeres: En cuanto al apoyo a las políticas de contratación, de promoción o de asesoría. 
- Seguridad e higiene en el trabajo: En cuanto a las políticas sobre el entorno laboral, precaución de accidentes, control médico o de alimentación.

\section{Perspectiva crítica}

Aunque en la actualidad se considera la RSC como un instrumento indispensable de las políticas corporativas, también, y no es menos cierto, que en los últimos tiempos se han detectado una serie de problemas que apuntamos para llamar la atención sobre ello; por un lado existen organizaciones que desconocen todo lo relacionado con la RSC, entre las cuales se halla una parte de las empresas públicas, ya que sigue despertado cierta desconfianza sobre sus implicaciones y consecuencias, por otro lado para algunas grandes empresas se ha convertido en una simple cosmética de la imagen.

Arceo Vacas (2004) describe el panorama de la RSC como negativo en cuanto a la eficacia, considerando que la mayor parte de éstas son más un gasto que una inversión, señalando la ausencia de investigación como una de las razones fundamentales de su ineficiencia. Continúa diciendo que para que ésta sea eficaz debe ser estratégica sino se seguirá identificando con la mera publicidad, con operaciones de marketing y con relaciones públicas modelo agente de prensa o publicity -siguiendo los cuatro modelos de relaciones públicas establecidos por Grunig y Hunt-. En este modelo el público no interesa y la empresa se limita únicamente a informar de aquello que puede aportar imagen a la empresa.

En esta perspectiva crítica se pone en tela de juicio las motivaciones reales que existen detrás de las prácticas filantrópicas, estas acciones de compromiso social son calificadas como truco publicitario y como una mala inversión ya que carecen del rigor de la investigación para conocer si se consiguen los efectos o impactos deseados, en este sentido la RSC se alejaría de la génesis de las relaciones públicas.

Pozas Pardo S., en el capítulo "El tránsito de la filantropía a la responsabilidad corporativa global. Un cambio necesario", en la obra de Arceo Vacas (2004), dirá sobre este punto:

Mientras la RSC no dé respuesta a las exigencias sociales y medioambientales reales, mientras las actividades empresariales sigan siendo controvertidas, mientras las empresas se nieguen a aceptar una legislación rigurosa, toda retórica sobre la RSC seguirá siendo vista con escepticismo, seguirán apareciendo como operaciones de cosmética y superficiales, con un lenguaje atractivo pero vacío, y seguirán despertando desconfianza. (pp. 242)

Coincidiendo con dicha afirmación concluimos que no son pocos los casos que han salido a la luz de empresas que parecían tener un claro objetivo ético con este tipo de estrategias, pero a la sazón se descubrió que no era el caso y que lo que se pretendía como fin último era un lavado de imagen. Esto, evidentemente, daña la imagen de todas aquellas otras que sí lo hacen con un objetivo filantrópico real. Aquí el papel de las relaciones públicas es capital a la hora de asesorar en materia de políticas y comportamientos éticamente estratégicos.

En relación con el desarrollo local, la crisis económica y financiera que llevamos padeciendo desde 2009 ha afectado, de manera contundente, a las políticas de empleo y 
desarrollo local. Aguado i Hernández J.A. (2013) afirma que en este contexto actual de crisis han surgido conductas irresponsables con ausencia de ética, pero que aun así la RSC puede convertirse en una herramienta que puede aportar nuevas alternativas al desarrollo local para escapar de la crisis a través del fomento de la integración y de las colaboraciones empresas-administraciones públicas. No obstante, advierte que este compromiso será efectivo cuando las empresas añadimos nosotros y administraciones públicas- tomen decisiones basadas en la sostenibilidad "desde un planteamiento integrado y estratégico atendiendo a la demanda de los grupos de interés" (p. 147) y no como una estrategia de maquillaje de imagen corporativa.

\section{RELACIONES PÚBLICAS EN LA ADMINISTRACIÓN PÚBLICA LOCAL Y RSC}

Una parte importante de los manuales de relaciones públicas que abordan los diferentes sectores donde se puede ejercer la RSC, hacen mención especial al ámbito de la Administración Pública, entre ellos se alude a la importancia de las relaciones públicas en la Administración pública local, de los cuales extraemos algunas referencias que nos parecen oportunas.

Oliveira, María da Luz y otros (1993) apuntan a que las estrategias de relaciones públicas que podemos aplicar no siempre serán las mismas porque depende del tipo de naturaleza, tamaño, situación, o momento en que se estén desarrollando las actividades de una institución pública, y nos dice:

Las actividades de relaciones públicas varían de una institución pública a otra, de una situación a otra, de una circunstancia a otra. En el tiempo y en el espacio las relaciones públicas cambian (...) de una situación buena a una situación de crisis, de una fase económica próspera a una fase de depresión (...) Para alcanzar los objetivos es necesaria mucha actividad, mucha acción y mucha práctica. Las funciones de un profesional de relaciones públicas en la administración pública son múltiples, no pudiendo describirlas todas en un libro de forma pormenorizada ( $p$. 116)

Lewis, Geoffrey en su capítulo "Public Relations in Local Government" de la obra de Howards I. y otros (1986), expone la idea de que un gobierno local, elegido por el pueblo para trabajar por el pueblo, ha escuchado su voz por lo consiguiente es importante que las autoridades locales hagan uso de las habilidades prácticas de las relaciones públicas para mantener la comunicación positiva con el pueblo. Esta función del profesional de relaciones públicas es de tal importancia que parece sensato que se ubique dentro de la estructura de la alta dirección de la Administración Pública local. Finalmente, hace un apunte que nos parece interesante, en lo que a la gestión del desarrollo local se refiere, y es que el relaciones públicas deberá explicar las políticas de desarrollo económico y social con la misma experiencia que si fuese el propio político; ponemos en consideración la importancia de esta idea porque el AEDL -Agente de Empleo y Desarrollo Local-, aun no siendo un político, sí deberá de transmitir y gestionar claramente las políticas que se vengan aplicando en dicho servicio, por lo que está realizando una función esencial de las relaciones públicas, y esta es: informar y comunicar buscando la comprensión y el entendimiento de los distintos públicos. 
Lloyd H. y Lloyd P (1988) manifiestan que dirigir una ciudad o municipio es muy parecido a dirigir una empresa, es cuestión de crear confianza con la comunidad y la función de las relaciones públicas es servir imparcialmente a la Administración local, independientemente del partido político que esté en ese momento en el poder. Nos dice:

El funcionario de relaciones públicas de la administración local está para servir al ayuntamiento, al contribuyente y a la prensa (...) hallará el desafío de establecer un equilibrio entre los tres, y necesita recabar información sobre asuntos complejos de tal forma que todos los públicos sean capaces de comprender lo que se pretende. Precisa introducir publicaciones eficaces, promocionar la región, promover reuniones, conferencias y exposiciones interesantes; diferentes medios de comunicación de modo que saque el mayor aprovechamiento posible; y finalmente, estar totalmente dispuesto a servir a la comunidad ( p. 39)

Por otro lado, Wilcox y otros (2012) sobre los gobiernos locales nos habla de la importancia de crear un flujo de información permanente porque el objetivo principal es informar a los ciudadanos sobre los servicios municipales y "ayudarles a que saquen el máximo partido de ellos" (p. 534), además de promocionarse a sí mismos para atraer nuevos negocios que promuevan el desarrollo económico y social de la zona. Uno de los apartados que nos parece relevante, y que menciona Wilcox, dentro del espacio de la gestión de relaciones públicas en la Administración Pública, son las relaciones institucionales consistentes en defender los intereses colectivos o de empresas ante las distintas Administraciones Públicas, éstas se basan en dos pilares: la gestión de conflictos potenciales y los grupos de presión.

Otro autor ya mencionado y que nos parece interesante señalar, por su aportación a este asunto, es Black, S. (1991) el cual nos viene a reafirmar las ideas expuestas hasta ahora, por los demás autores, confirmando el hecho de que el gobierno local es una responsabilidad social compartida que requiere el trabajo de todos y que la gestión de las relaciones públicas proporcionan el medio más efectivo para alcanzar esa cooperación; destacando como principal objetivo el potenciar la concienciación ciudadana de despertar interés activo por las gestiones de su gobierno local. Black formula cuatro objetivos principales de relaciones públicas en el gobierno local (pp. 191-192):

1. Mantener informado al pueblo de la política municipal y de las actividades cotidianas.

2. Dar al público la oportunidad de expresar sus puntos de vista sobre nuevos proyectos, antes de que se tomen las decisiones.

3. Educar al pueblo sobre la manera en que funciona el sistema de gobierno local e informarle sobre sus derechos y responsabilidades.

4. Promover el sentimiento de orgullo cívico.

En definitiva, podemos observar que estos autores destacan una idea que ha de presidir las relaciones públicas de la Administración local, y que éstas han de conseguir que en la mente del ciudadano esté presente y sea consciente de la gestión diaria del administrador local, entre la que destacamos la gestión estratégica de desarrollo local y turismo sostenible en el propio municipio. 


\section{TURISMO Y DESARROLLO LOCAL SOSTENIBLE}

El concepto de desarrollo sostenible fue introducido por la Comisión Mundial sobre Medio Ambiente y Desarrollo en el llamado "Informe de Brundtland" en 1987, definiéndolo como "desarrollo que satisface las necesidades del presente sin comprometer la capacidad de las generaciones futuras para satisfacer sus necesidades propias". (p.1, Cap. 1.1.) Más tarde, en 1993, en la Conferencia Euromediterránea sobre Turismo y Desarrollo Sostenible, la Organización Mundial del Turismo (OMT) en un documento titulado "Tourism the year 2000 and beyond qualitive aspects" definió el concepto de Turismo Sostenible:

El Turismo sostenible atiende a las necesidades de los turistas actuales y de las regiones receptoras y al mismo tiempo protege y fomenta las oportunidades para el futuro. Se concibe como una vía de cara a la gestión de todos los recursos de forma que puedan satisfacerse las necesidades económicas, sociales y estéticas, respetando al mismo tiempo la integridad cultural, los procesos ecológicos esenciales, la diversidad biológica y los sistemas que sostienen la vida. (p. 23)

Vargas Martínez, E.E., y otros (2011) argumentan que en este proceso del origen del término existe un momento álgido que se alcanzó en 1995 en Lanzarote cuando se redacta la "Carta del Turismo Sostenible", y se establecieron los dieciocho principios que guiarían a todos los actores del turismo hacia la sustentabilidad. Bajo el paraguas de este concepto hay dos factores que resaltar en su interpretación, y son la perspectiva de conservación y el equilibrio entre las dimensiones social, económica y ecológica y la aparición, a posteriori, de regulaciones y políticas ambientales.

Posteriormente serán publicados otros documentos importantes a este respecto como la "Guía Práctica para el Desarrollo y Uso de Indicadores de Turismo Sostenible" publicado en 1997 por la OMT. En ese mismo año surge la Declaración de Berlín sobre Diversidad Biológica y Turismo Sostenible, cuyo objetivo es adaptar los principios del Convenio de Diversidad Biológica, firmado en Río en 1992, a la actividad turística. Y 2002 fue declarado el Año Mundial del Ecoturismo.

Aparecerá un amplio debate sobre la sustentabilidad que, dicen Vargas Martínez y otros (2011), se concentrará especialmente en el uso de los recursos naturales pero, sin embargo, existen otros recursos importantes como son los socioculturales lo que lleva a ampliar el significado y a dedicar más estudios a otro tipo de recursos, ya que es un término en constante evolución, además de albergar cierta ambigüedad significativa.

Relacionado con el ámbito del turismo, tanto en el campo académico como profesional, se lleva tiempo debatiendo sobre el concepto de "turismo sostenible" o "sustentable", porque se trata de un concepto complejo que abarca factores de índole ecológico, cultural y socioeconómico, que implica gestionar el turismo en base a unas estrategias de equilibrio y armonía entre turismo y medio ambiente, no siempre fácil de alcanzar. En ocasiones debido a la propia fragilidad del ecosistema y de los recursos disponibles en el destino, o por la falta de sensibilidad y sensatez por parte de los actores sociales, cómplices en la planificación y gestión que ha llevado a innumerables casos con consecuencias de daños irreparables en el medio ambiente. 
Por lo tanto, la actividad turística sustentable se presenta como una opción, una oportunidad para el desarrollo local y se manifiesta como una vía de mejora de la calidad de vida de la población local ya que respeta el medio natural y la cultura local, a la vez que impulsa los recursos endógenos del propio municipio. El aprovechamiento de los recursos naturales y culturales del municipio de forma responsable es la idea que subyace en el desarrollo del turismo sostenible.

\section{PROPUESTA DE GESTIÓN INICIAL: TURISMO SOSTENIBLE, DESARROLLO LOCAL, RELACIONES PÚBLICAS Y RESPONSABILIDAD SOCIAL CORPORATIVA}

Aunque el concepto de "responsabilidad social corporativa" -RSC- ha sido abordado desde el enfoque empresarial, ya que su génesis arranca en este contexto, hemos realizado una aproximación desde la disciplina de las relaciones públicas con el objetivo de interconectar responsabilidad social corporativa, desarrollo local y turismo, puesto que las autoridades públicas deben tener en cuenta prácticas socialmente responsables en la implementación de las políticas de desarrollo en su propio beneficio y en el de la comunidad.

El turismo es un fenómeno social de nuestros tiempos que lleva una larga etapa experimentando grandes cambios en los países desarrollados. Han surgido nuevos modelos de turismo, paralelos al turismo tradicional, que requieren de una adaptación rápida por parte de empresas e instituciones públicas. A nivel local el turismo ha adquirido mayor importancia, al tiempo que lo ha hecho también a nivel global, dándose el fenómeno de la glocalización, los proyectos turísticos de desarrollo local han tenido mayor protagonismo en las últimas dos décadas, iniciados con el plan FUTURES, hecho que se ha venido potenciando a lo largo de los últimos años. Es por ello que la labor del AEDL, en este sector, se vio incrementada notablemente, dentro de las políticas de desarrollo local.

Milio y Cabo (2000) apuntan que el principal objetivo de las relaciones públicas turísticas es la creación de una imagen buena que resalte los valores más positivos de la empresa o destino turístico. A través de los distintos medios y soportes informativo-comunicativos vendemos imaginarios y éstos son fundamentales para incrementar o mantener el número de visitantes de un destino, así pues, la imagen que podemos tener de un destino va a condicionar la elección del destino y del comportamiento posterior del turista. Riemer (1990), citado por Álvarez Sousa (2009), considera que el sistema turístico está basado en la producción y distribución de sueños imaginarios sobre un determinado lugar, sistema que intenta vender imaginarios a los turistas que quieren escapar del mundo cotidiano. La promoción de un destino, dice Álvarez Sousa, se puede realizar por distintos medios: a través de la publicidad fundamentalmente en televisión, la publicidad en vallas publicitarias y otros soportes, el patrocinio de eventos, las ferias, actuaciones educacionales y también las relaciones públicas.

García Iglesias, D. (2010), en un estudio realizado sobre relaciones públicas y turismo para el Ministerio de Turismo de la Habana en Cuba, resalta la importancia de la estrategia de relaciones públicas en el intento de promocionar el turismo, y tomamos como ejemplo la siguiente afirmación: 
La organización turística (pública o privada) tiene que trabajar intensamente con los ciudadanos residentes en los destinos, para la elevación de su cultura del turismo y la hospitalidad. La idiosincrasia de estos individuos es uno de los principales móviles dentro del proceso de organización de un periplo turístico, cuestión que no debiera ser aprovechada únicamente por el discurso publicitario (con sus consabidas edulcoraciones y figuras hiperbolizadas) sino también por las relaciones públicas pero desde una perspectiva más estratégica, desde la cual se contribuya a hacer de los anfitriones una parte activa del amplio concepto de producto turístico" (pp. 517)

Ambos enfoques, dirá García Iglesias, la publicidad con un objetivo de beneficio económico y las relaciones públicas con un objetivo de beneficio social, no tienen que estar reñidos aunque es difícil encontrar el punto de equilibrio entre los dos, especialmente en el turismo donde la idea de lucro está muy interiorizada y mucho más legitimada que cualquier otra. Si se realizan las estrategias comunicacionales únicamente desde la óptica comercial, la organización y gestión de las actividades turísticas se centran en el intercambio exclusivamente comercial, ello restringe a las organizaciones e instituciones, en este caso a las AEDLs, sus posibilidades de promoción integral con el objetivo de un desarrollo de éxito en el área local.

Sin menospreciar los procesos comerciales necesarios para el posicionamiento del producto turístico desde el marketing o la publicidad, hemos de reconocer el beneficio que nos aporta el uso de las estrategias y técnicas que nos ofrece las relaciones públicas, respetando los objetivos y funciones específicas de cada una de ellas. Éstas, y esto hay que tenerlo muy claro, multiplican el impacto del resto de las acciones y actividades de promoción, este hecho ayuda a potenciar el factor de la diferenciación respecto a la competencia, ya que las relaciones públicas parten de una visión más global del conjunto de la institución. Dirán Al Ries y Laura Ries (2003) que la publicidad ha de estar supeditada a las relaciones públicas, en el sentido de que son una continuación cuando las relaciones públicas han consolidado la marca.

Aguirre, B. y Hernández, C. (1991) definen las relaciones públicas turísticas como aquellas técnicas encaminadas a crear una imagen correcta y a dar reconocimiento a la personalidad pública de las organizaciones, así como establecer nuevos sistemas de comunicación social en busca de una comprensión. Dicen textualmente, "Las relaciones públicas han de crear, dar a conocer y prestigiar la imagen de la empresa, marca, producto o servicio y de los individuos que la integran" (p. 57). Concluyen afirmando que, el servicio de relaciones públicas, se puede aplicar tanto a la Administración Pública como a la empresa privada y que sirve de canal de comunicación entre los diferentes públicos, principal función de éstas.

Álvarez Sousa, A. (2009) incide en la idea de que si proyectamos una imagen de turismo sustentable responsable -basado en la responsabilidad social corporativa- obtendremos turistas respetuosos con el medio ambiente porque los destinos que se preocupan por crear una imagen (valor simbólico) para ser visitados por turistas con un comportamiento ético, tienen más posibilidades de ser visitados por turistas responsables y reflexivos.

En el llamado Libro Verde, elaborado por la UE en 1998, quedó recogido el concepto que tuvo el apoyo del Parlamento Europeo, el cual propuso incorporar el término de "responsabilidad social" de la empresa a otros ámbitos de competencia de la UE, en concreto en el tema de 
financiación de medidas sociales y regional. Este término lo definió la Comisión Europea, a través del Libro Verde, como aquél tipo de responsabilidad donde "las empresas y otras entidades deciden voluntariamente integrar las preocupaciones sociales y ecológicas en sus actividades comerciales y en las relaciones con sus interlocutores" (Farto López, 2006: 160).

En esta misma obra, Farto López acude a la definición de la RSC, propuesta por la World Business Council for Sustainable Development (WBCSD) (1998), definición que nos parece muy interesante desatacar por estar integrada en el contexto del desarrollo local que dice así: "La decisión de la empresa y otras entidades de contribuir al desarrollo sostenible, trabajando con sus empleados, sus familias y la comunidad local, así como con la sociedad en su conjunto, para mejorar su calidad de vida" (p. 172)

De todo lo expuesto se desprende que estamos hablando de un concepto intrínsecamente ligado a la existencia, dentro del territorio del capital social, de gobernanza de calidad y amplio consenso entre los actores sociales implicados sobre los objetivos de la RSC. El desarrollo local es producto de la coordinación y la cooperación entre todas las partes interesadas, del trabajo en red y el aprendizaje social, que a su vez propicia la regeneración y gestión de aquellos factores de los que se retroalimenta para producir desarrollo local sostenible.

Las técnicas comunicativas de relaciones públicas, y la estrategia de la RSC, adquieren un papel esencial en un contexto de mayor concienciación medioambiental, mayor competitividad y mayor oferta turística. Al hablar de turismo, hablamos de un servicio intangible que vende sensaciones, emociones, conocimiento y experiencia, y las relaciones públicas están más próximas a dichos valores. En la obra de Cebrían Abellán (2008), Molina Collado en el capítulo "Comunicación e imagen de productos y destinos turísticos" (pp.241-249), ya advierte que existe una clara evidencia en la dificultad de comunicar los atributos intangibles y convertirlos en tangibles con el objetivo de que éstos puedan tener mayor impacto en la conciencia de los sujetos. De ahí que reconfirmamos el papel tan sumamente importante de las relaciones públicas.

\section{CONCLUSIONES}

Resumiendo, en forma de esquema, el proceso de dinámica de gestión relacional de la RSC en el desarrollo local y turismo sostenible que proponemos como línea de trabajo sería el siguiente:
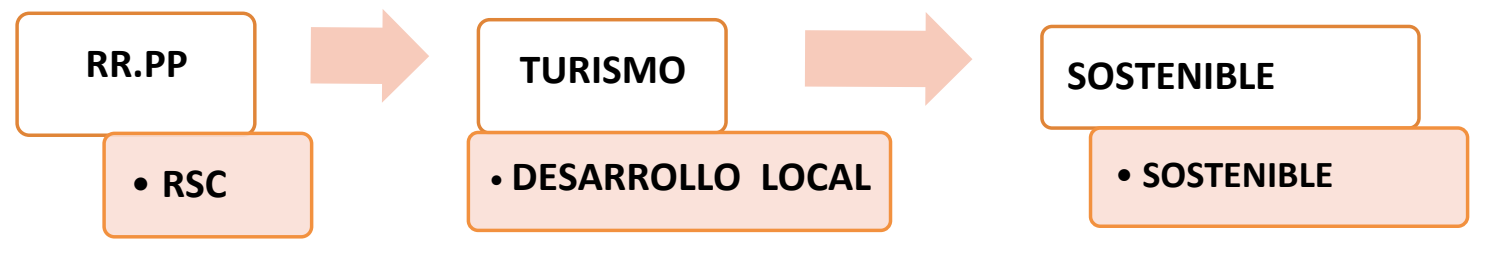

Figura 2: Estrategia de trabajo en el desarrollo local y el turismo sostenible, basado en el enfoque de las relaciones públicas y de la responsabilidad social corporativa. Elaboración propia. 
Las interrelaciones que se pueden establecer entre estas áreas de conocimiento y usos de estrategias, en el contexto de la gestión de actividades turísticas, nos permiten determinar unas líneas claras de trabajo.

Podemos observar en la figura 2 una serie de vínculos estratégicos que pueden contribuir a la mejora de la estrategia comunicacional de las políticas de desarrollo local - desde la AEDL-, de forma que el profesional que ejerza la labor, dentro de la agencia, pueda afrontar sus funciones comunicativas con una visión y conocimientos más específicos sobre los beneficios sociales que puede aportar la aplicación de las relaciones públicas, así como, a largo plazo, repercutir y traducirse en beneficios de índole económica. Por lo tanto, el AEDL se puede convertir en el sujeto promotor de las políticas de desarrollo local en el área de turismo, desde la filosofía de las relaciones públicas.

Existen una serie de convergencias, siendo el desarrollo local sustentable, y por añadidura el turismo sostenible, el que mayor vinculación teórica-práctica presenta con el enfoque táctico de las relaciones públicas. El beneficio social que aporta la gestión relacional ayuda a impulsar el desarrollo local de manera más exitosa -la búsqueda de las relaciones de confianza, reciprocidad, comprensión mutua, cooperación y el sentimiento de pertenencia-, sientan las bases para promover el desarrollo sostenible, a la par que el turismo también sostenible. Todo ello es posible si se gestiona desde la óptica de la buena gobernanza, siempre desde la gestión política y estratégica de la responsabilidad social corporativa -uso de las buenas prácticas, ética, transparencia en la gestión, búsqueda de la comprensión de los públicos, la negociación equilibrada y la resolución de conflictos- y de interacciones de confianza.

El capital simbólico, básico para la consecución de objetivos relacionados con la imagen de marca de un destino, y sus interrelaciones con el desarrollo local sostenible, así como el turismo sostenible $y$, desde la filosofía de las relaciones públicas, tendrán mayores oportunidades de consolidación para, a posteriori, trabajar otras estrategias comunicativas de refuerzo, una vez realizada su labor la función de relaciones públicas. A su vez el turismo sostenible y responsable habrá hecho hincapié en la conservación y recuperación del medio natural y cultural, no como una simple estrategia puntual de maquillaje de RSC sino como política de continuidad y de concienciación colectiva de todos los actores sociales implicados.

\section{REFERENCIAS}

AGUADO i HERNÁNDEZ, JULI ANTONI (2013) "La responsabilidad social corporativa y el desarrollo local: una aproximación en tiempos de crisis". Rev. De Investigación Social Prisma Social. Revista de Ciencias Sociales, no 10, nov 2013. Sección temática: Responsabilidad Social Corporativa, ¿oportunidad o crisis?, pp. 119-156.

http://www.isdfundacion.org/publicaciones/revista/numeros/10/secciones/tematica/pdf/04rsc-desarrolo-local.pdf

AGUIRRE, B. y HERNÁNDEZ, C. (1991) El lenguaje del turismo y de las relaciones Públicas. Española de Librería S.A. Sexta edición. 
ÁLVAREZ SOUSA, A. (2009) Sociología del turismo. Udima (Universidad a Distancia de Madrid). Edita Centro de Estudios Financieros. (p 212). Fuente citada: Riemer, G. (1990) "Packaging Dreams", Annals of Tourism Research, no 17, pp. 501-512.

ARCEO VACAS, J. L. (2004) - Coord.- Las Relaciones Públicas en España. Editorial Mc Graw-Hill S.A. México. Primera Edición.

BALAGUER FRANCH. Mạ R., FERNÁNDEZ IZQUIERDO, Mạ A. y MUÑOZ TORRES, Ma J. (2007) La responsabilidad social de la empresa. Relación entre la performance social, financiera y bursátil. Generalitat Valenciana, Consellería d'empresa. Universitat i Ciència, pp. 25-27. Fuente citada: Carroll (1991) The pyramid of corporate social responsability: towards the moral management of organisational stakeholders. Business Horizons, vol. 34, no 4, pp. 34-48.

BERNAYS L. E. L. (1990) Los últimos años. Radiografía de las Relaciones Públicas 1956-1986. ESRPPPU. Barcelona, pp. 23-24; 80.

BLACK, SAM (1991) Las relaciones públicas, un factor clave de gestión. Editorial Hispano Europea. Barcelona

BLACK, SAM (1993) ABC de las Relaciones Públicas. Ediciones Gestión 2000 S.A., Barcelona

CEBRIÁN ABELLÁN. F. -Coord.- (2008) Turismo rural y desarrollo local, Capítulo "Comunicación e imagen de productos y destinos turísticos" de Molina Collado, A. (241-249). Ediciones de la Universidad de Castilla-La Mancha. Sevilla, Cuenca

COMISIÓN MUNDIAL SOBRE MEDIO AMBIENTE Y DESARROLLO (1987) Informe Brundtland. http://www.un-documents.net/wced-ocf.htm

FARTO LÓPEZ, J. (2006) Desarrollo Local sostenible en Territorios inteligentes y responsables. Documenta. Instituto Europeo para la Formación y el Desarrollo. Cantabria.

FRASER, P. S. (2002) Teoría y práctica de las relaciones públicas. Editorial Pearson Educación. Madrid. Octava edición.

GARCÍA IGLESIAS, DOGOBERTO (2010) Relaciones públicas aplicadas a contextos turísticos. Aproximaciones al fenómeno desde la emergencia de un enfoque integrador. Estudios y perspectivas en Turismo. Documentos especiales. Editado por Ministerio de Turismo de la Habana. Cuba. http://www.scielo.org.ar/pdf/eypt/v20n2/v20n2a13.pdf

GRUNIG, JAMES. E. y HUNT, TODD. (2000) Dirección de relaciones públicas. Editorial Gestión 2000. Barcelona. Primera edición en castellano. Fuente citada: Preston Lee E. y James E. Post Private Management and Public Policy: The Principle of Public Responsibility (Englewoods Cliffs, N.J. Prentice-Hall, 1975).

HOWARD I., WILFRED, M.A., M. CAM. F.I.P.R. (1986) The Practice of Public Relations. Heinemann London Ltd. London. Second edition. Fuente citada: Lewis, Geoffrey (1986) "Public Relations in Local Government, Chapter 8, pp. 150-159. 
LLOYD, HERBERT. y LLOYD P. (1988) Relaçôes Públicas. As técnicas de comunicacçâo no desenvolvimento da empresa. Colección Biblioteca de Gestô Moderna. Editorial Presença. Lisboa.

MILIO BALANZÁ, ISABEL y CABO NADAL, MÓNICA (2000) Comercialización de productos y servicios turísticos. Hostelería y Turismo. Editorial Paraninfo S. A. Madrid.

NOGUERO, A. (1990) Relaciones Públicas e industria de la persuasión. Colección Comunicación y Relaciones Públicas. PPU. Barcelona

OLIVEIRA, M.L., JOÂO PAIS, M. y GIL CABRITO, B. (1993) Relaçôes Públicas. Texto Editora. Porto.

PARÉS i MAICAS (1994) La nueva filantropía y la comunicación social: mecenazgo, fundación y y patrocinio. Colección Comunicación y Relaciones Públicas. PPU. Barcelona.

RIES, AL y RIES, LAURA (2003) La caída de la Publicidad y el auge de las Relaciones Públicas. Ediciones Urano S.A. Barcelona.

VARGAS MARTÍNEZ, E.E, CASTILLO NECHAR, M. y ZIZUMBO VILLARROEL, L. (2011) "Turismo y sustentabilidad. Una reflexión epistemológica". Revista Estudios y Perspectivas en Turismo. Vol. 20, pp. 706-721. http://www.estudiosenturismo.com.ar/PDF/V20/N03/v20n3a10.pdf

WEIL, PASCALE (1992) La comunicación global. Editorial Paidós Comunicación. Barcelona. Segunda edición.

WILCOX DENNIS .L, CAMERON GLEN T. Y XIFRA, JORDI (2012) Relaciones Públicas. Estrategias y tácticas. Addison Wesley en su sello editorial autorizado de Pearson Educación S.A. Madrid. Décima Edición ampliada. 